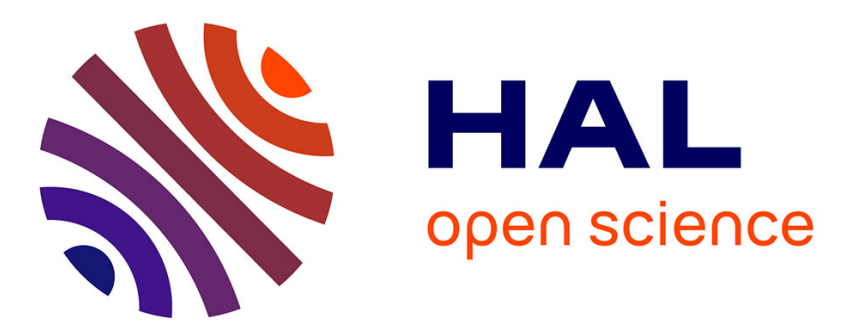

\title{
Digital to analog conversion methodology applied to self-mixing displacement signals
}

Antonio Luna Arriaga, Francis Bony, Thierry Bosch

\section{To cite this version:}

Antonio Luna Arriaga, Francis Bony, Thierry Bosch. Digital to analog conversion methodology applied to self-mixing displacement signals. 10TH INTERNATIONAL CONFERENCE ON VIBRATION MEASUREMENTS BY LASER AND NONCONTACT TECHNIQUES - AIVELA 2012, Jun 2012, Ancona, Italy. pp.125-131, 10.1063/1.4730550 . hal-00716538

\section{HAL Id: hal-00716538 \\ https://hal.science/hal-00716538}

Submitted on 10 Jul 2014

HAL is a multi-disciplinary open access archive for the deposit and dissemination of scientific research documents, whether they are published or not. The documents may come from teaching and research institutions in France or abroad, or from public or private research centers.
L'archive ouverte pluridisciplinaire HAL, est destinée au dépôt et à la diffusion de documents scientifiques de niveau recherche, publiés ou non, émanant des établissements d'enseignement et de recherche français ou étrangers, des laboratoires publics ou privés. 


\title{
Digital to Analog Conversion Methodology Applied to Self-Mixing Displacement Signals
}

\author{
Antonio Luna Arriaga ${ }^{1,2}$, Francis Bony ${ }^{1,2}$ and Thierry Bosch ${ }^{1,2}$ \\ ${ }^{1}$ CNRS, LAAS, 7 avenue du colonel Roche, F-31400 Toulouse, France \\ ${ }^{2}$ Univ de Toulouse, INP, LAAS, F-31400 Toulouse, France
}

\begin{abstract}
An adaptive algorithm has been designed to treat interferometric self-mixing signals for weak and moderate laser feedback. With regard to the creation of an embedded displacement sensor, the reconstruction process is inspired from the interpolation feature found in digital-to-analog converters. By using an adaptive filter as the central process, the algorithm presents a high potential of parallelism to meet timing restrictions. A validation for different signal scenarios was carried out. The obtained precision and ease of maintainability enlightens its capabilities to be coupled with other signal processing methods to produce better system reliability.
\end{abstract}

Keywords: Adaptive algorithm, signal reconstruction, optical feedback, displacement measurement

PACS: $42.60 .-\mathrm{v}, 06.30 . \mathrm{Bp}$

\section{INTRODUCTION}

Self-mixing (SM) interferometry for metrological purposes has been studied with more interest in the last ten years as an inexpensive and auto-aligned optical sensing technology [1]. The reduced number of components required for its setup and the precision achieved with this technique, place it as a prominent candidate for development of embedded applications. In the field of displacement measurement the SM signal processing is rather complex due to the strong dependance to the target reflectivity [2]. Efforts are still carried to consolidate it as a reliable instrumentation scheme.

In a classical displacement reconstruction algorithm [3], the optimization procedure launched to reach more precision works over a full signal acquisition and requires several cycles to converge. Hence, this algorithm is not suitable for real time implementation, although their main objective was to enlighten the precision and feasibility of their method. Accuracy improvement has been reported by using an electro-optic modulator (EOM) which provides a better resolution to detect the shape of SM signals [4]. However the fact of adding external optics breaks the premise of conceiving a low-cost sensor. A recent algorithm has been proposed in [5] providing a solution to discriminate the error arising from the different reflective index of the materials under measure and their media. In that case, the study was oriented to the robustness achieved and not in the implications of the real time elaboration.

The self-mixing interferometer setup is presented in Fig.1. It only requires a laser diode (LD) as a source, a collimating lens and a photodiode (PD) to measure the optical output power (OOP) of the laser beam. The processor unit required to interpret the incoming SM signals, provides information about the target's displacement with a precision inferior to half-wavelength. When the LD is focused on a moving target, a small portion of the emitted beam is back-reflected by the target's surface into the laser cavity. This produces a variation of power and frequency in the emission stage. The equation describing the power monitored as seen by PD is

$$
P(t)=P_{0}\left[1+m . F\left[\frac{4 \pi D(t)}{\lambda_{F}(t)}\right]\right] .
$$

The emitted optical power from the laser without back-reflection is $P_{0}$. The modulation index for the laser diode is $m$. The target displacement is $D(t)$. The emission wavelength subject to optical feedback is $\lambda_{\mathrm{F}}$, and $F$ is a cosine function related to the feedback coupling factor $(\mathrm{C})$, used to characterize the shape of the signal when the internal oscillation of the laser is affected. When the target approaches to the source, SM signals exhibit a certain tilt. It is mirrored when the target goes in the opposite sense [2]. SM features can be compared to classical Michelson interferometers, however an important difference is that SM phenomenon occurs in the semiconductor active medium and not in the free space.

In order to dimension a system for an embedded usage, a time constraint must be imposed based on the requirements specification. Besides, the algorithm characterization provides knowledge of the needed resources for the target architecture. If we take as example two different applications from recent literature: (a) The monitoring of independent 


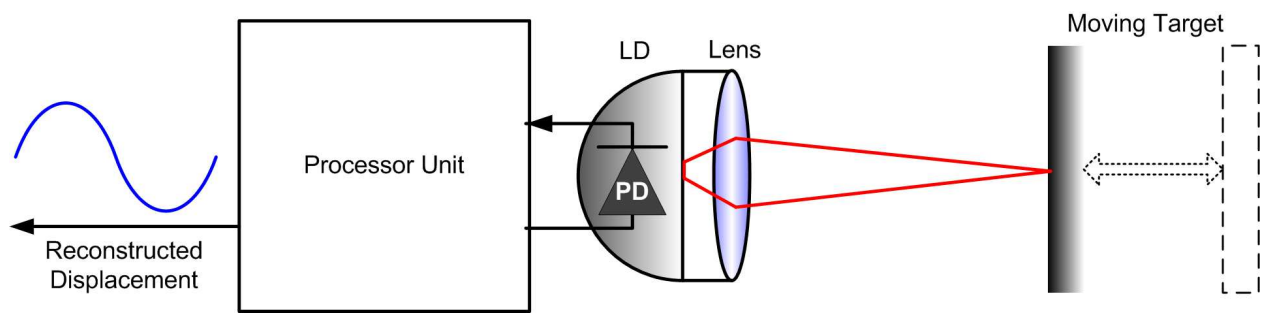

FIGURE 1. Self-mixing setup.

displacement of individual surface portions in an ablation drilling process [6], and (b) a prototype to estimate the motion of a human chest wall [7]. One can realize that there are different reflection coefficients generating a particular SM signal shape and thus, the difficulty of modeling each system for an optimal implementation.

The algorithm proposed in this paper, named (CNA) is inspired from the well known First-Order-Hold (FOH) interpolator model used in some Digital-to-Analog Converters (DAC) [8]. It aims to provide a consistent displacement reconstruction and an ease of system resources estimation. It is intended to be coupled with other algorithms dealing with the detection of the SM signals, face to changes of $\mathrm{C}$ parameter and the correction of degradation artifacts over the signal like the speckle and fringe loss. The focus is on an efficient implementation and a high degree of maintainability in order to be synthesized for different processor architectures.

\section{RECONSTRUCTION PROCESS}

\section{Overview}

Consider Fig. 2 to illustrate the proposed method: The SM signal is digitized and fed into a detector system. It helps to transform the complex SM shape to a train of pulses corresponding to the temporal maxima and minima of each phase transition. From literature [2] we know that each SM fringe corresponds to a target displacement of $\lambda / 2$, so this value is multiplied by the output of the transition pair (TP) block. On the other hand, there is a module counting the number of samples $(\mathrm{T})$ between two transitions needed to command the generation of a normalized triangular window (TRI). Depending on the TP values the zone detector module selects an upsample procedure. Each upsampled pair is filtered with TRI and thus, produces an interpolated segment as in a classical FOH model. The linear model approach for SM analysis was proposed by [9]. That lead us to privilege this interpolation scheme instead of the conventional sinc interpolation.

The transitions detected at the input are not equally spaced, so they can be interpreted as belonging to an irregular sampling of the calculated displacement. A pure DAC interpolation is not feasible due to aliasing phenomenon. While sophisticated reconstruction methods from non uniform samples are an active research in signal processing [10], they are mainly based on the use of time-invariant low pass filtering. In the case of SM signals a static temporal input

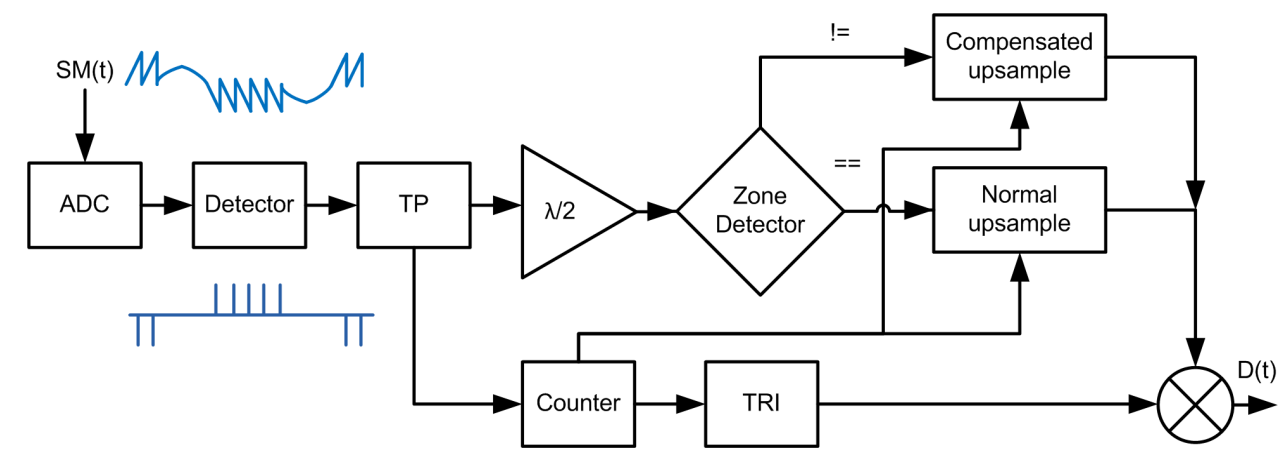

FIGURE 2. Block diagram for displacement reconstruction. 
cannot be guaranteed. To privilege the simplicity of calculations and the integration into a hardware-software system, the essence of the DAC integrator mechanism is kept as the heart of this algorithm.

\section{Formulation}

The TP vector is composed by the present and previous detected transitions $\operatorname{tr}(N)$ and $\operatorname{tr}(N-1)$ respectively, where $N=0,1,2, \ldots$ is the number of accumulated fringes detected. It is increased for positive fringes and decreased for negatives. TP can be represented as $[\operatorname{tr}(N-1) \operatorname{tr}(N)]$.

The signal $x(n)$ to convolve, is the resulting vector of upsampling TP by the factor $T$ as denoted by expression (2). On every new fringe arrival, TP is updated as well as the $T$ factor.

$$
x(n)= \begin{cases}T P(n / T), & n=1,2, \ldots, T \\ 0, & \text { otherwise. }\end{cases}
$$

The convolution kernel $h(k)$ gets a size $2 T$ as established by Shannon's sampling theorem. Its shape is TRI, corresponding to the impulse response of a delayed FOH interpolator [8]

$$
h(k)=(1 / T) T R I((k-T) / T) .
$$

The convolution of (2) and (3), results in the interpolated segment $\left(\mathrm{x}_{\mathrm{FOH}}\right)$ denoted by

$$
x_{F O H}(n)=\sum_{k=0}^{T} h(k) x(n-k) \text {. }
$$

This equation is graphically understood with the example of Fig.3, where a segment of two upsampled negative transitions is represented.

From literature [2] [3], it is known that target displacement can be calculated by
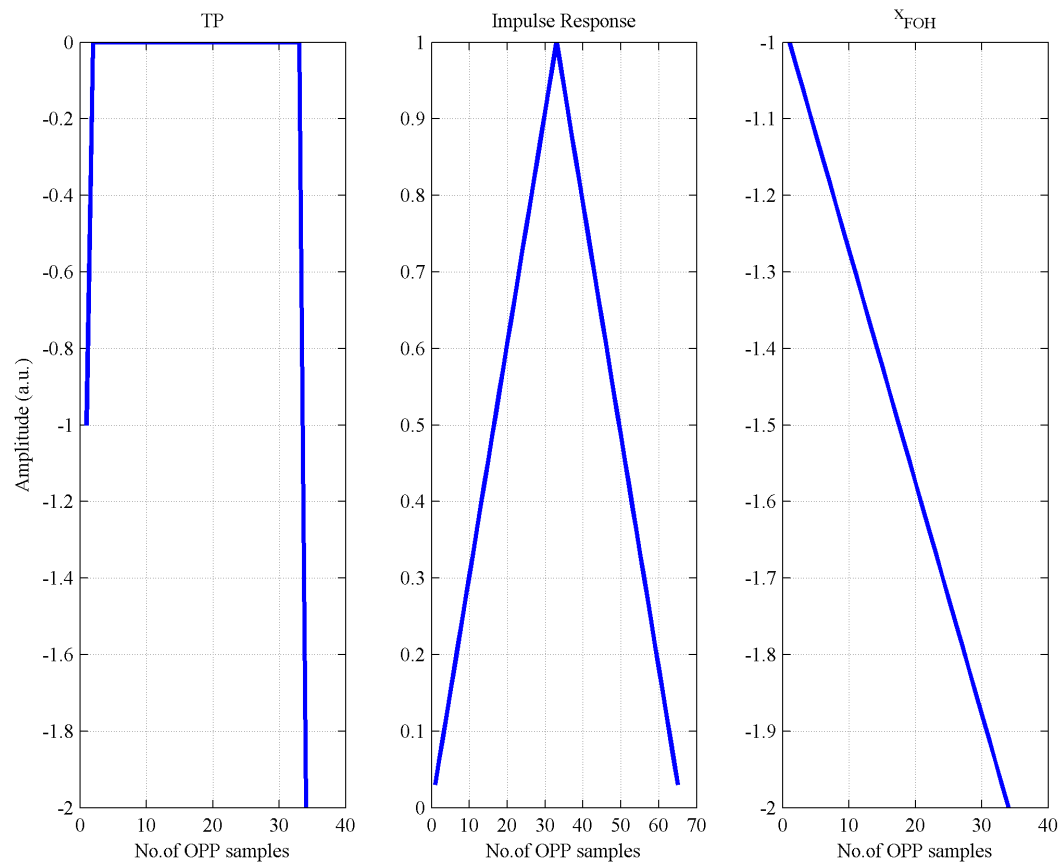

FIGURE 3. Representation of the generation of an interpolated fringe segment by convolution. 


$$
D(t)=N \frac{\lambda_{0}}{2}+\varepsilon
$$

where $\lambda_{0}$ is the laser diode wavelength without back-reflection. The excess fringe ' $\varepsilon$ ' is the point where the displacement changed direction. It helps to provide the precision inferior to $\lambda_{0} / 2$. Since the interpolated segments keep track of the accumulated transitions, the reconstructed displacement for our algorithm is

$$
D(n)=x_{F O H}(n) \frac{\lambda_{0}}{2}
$$

The heart of the algorithm is the convolution, which is one of the most well understood algorithms in signal processing domain. It can pass from a complexity $O\left(n^{2}\right)$ to $O(n \log n)$ with no big effort or even be implemented entirely into hardware [11]. In consequence, optimized implementations can be analyzed according to particular needs.

\section{Algorithm Details}

\section{Signal Detection}

Figure 4 shows the model of SM signals at two different laser feedback conditions, belonging to the same displacement. The weak back-scattered signal presents a sinusoidal shape, while the moderate regime offers a sawtooth like signal. A sawtooth shape is typically treated by implementing a high pass filter and a threshold comparison in order to extract the phase transitions. They are set positive for one direction of the target movement and negative for the opposite direction. SM shape obeys a non-linear behavior and as mentioned earlier, its analysis is adapted according to the given application. In order to validate our algorithm, we work with with a preprocessed vector of transitions. The discussion of the different methods for fringe detection at different $\mathrm{C}$ values is out of the scope of this work.
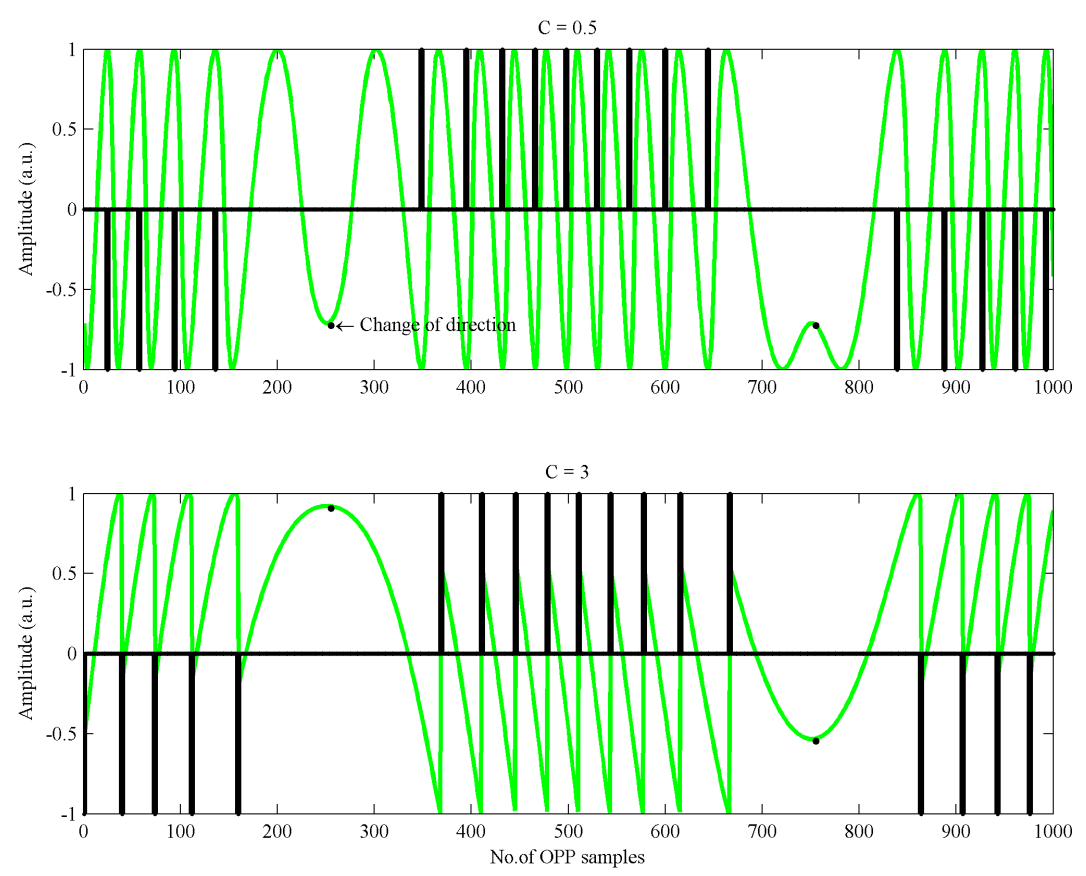

FIGURE 4. Modeled SM signals for a displacement of $5 \lambda$ with different feedback levels, alongside its detected transitions. 

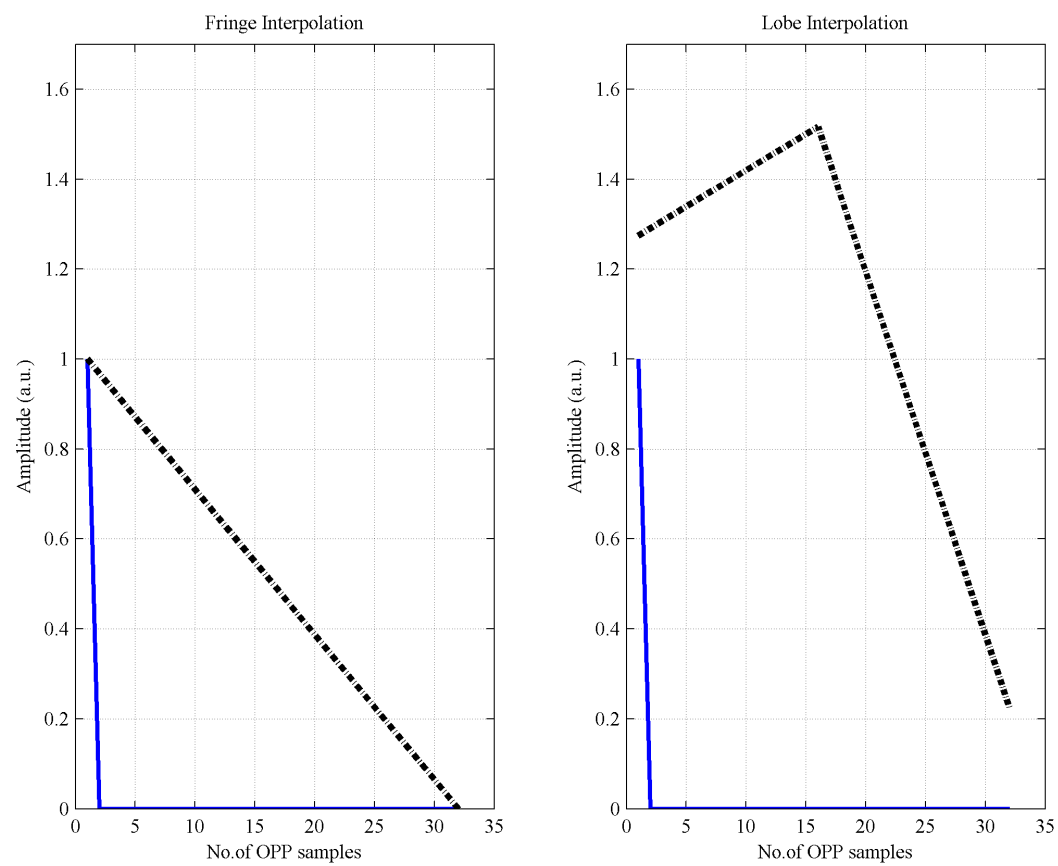

FIGURE 5. Comparison of a normal interpolation in the fringes zone and a compensated one for the lobes zone.

\section{Segments Interpolation}

The transitions vector is fed by pairs to TP module, if they share the same sign (positive or negative), then are assigned to a fringes zone. Conversely, when the target changes its direction there is a TP with a positive value and a negative value or vice-versa. This is assigned as a lobe zone. For the fringes zone, TP vector is simply upsampled by the current $\mathrm{T}$ counted samples. When reconstructing the lobe zone, TP vector is compensated by adding a value in the middle of TP vector, allowing the segment to bump providing more resolution to the signal shape. The precision achieved in (5) relies thus on this compensation.

Figure 5 shows in dotted lines the result of a linear interpolation for a TP segment in the two defined zones. The offset observed in lobe section degrades the shape reconstruction but it can be corrected by a smoothing filter to provide a clean signal at the output.

\section{Algorithm Validation}

The relevance and precision of the algorithm has been validated for different signal scenarios. In Fig. 6 we show the displacement reconstruction from the transitions of Fig. 4 for weak and moderate feedback. It can be seen that in both cases the periodic sinusoidal movement if retrieved by interpolating every two transitions. Hence, this is the real time constraint to consider when developing a system using this algorithm.

The error between the original displacement and the reconstructed signal is calculated from the sample to sample difference, the maximum error of about $250 \mathrm{~nm}$ shows that the algorithm doesn't follow a perfectly smooth sinusoidal displacement. This is due to the inherent segment by segment treatments. However in displacement measurement we are mainly concerned by the amplitude of the reconstructed signal as they provide us the displacement information of the target. Moreover, since the reconstructed displacement is in digital format an smoothing filter can be performed without penalize resources before taking out signal.

Finally we acquired a real signal with a LD from Hitachi (HL7851G) with $\lambda=785 \mathrm{~nm}$. The moving target was a piezoelectric transducer with nanometric resolution [12], configured to produce an harmonic displacement of $2.25 \mu \mathrm{m}$ 

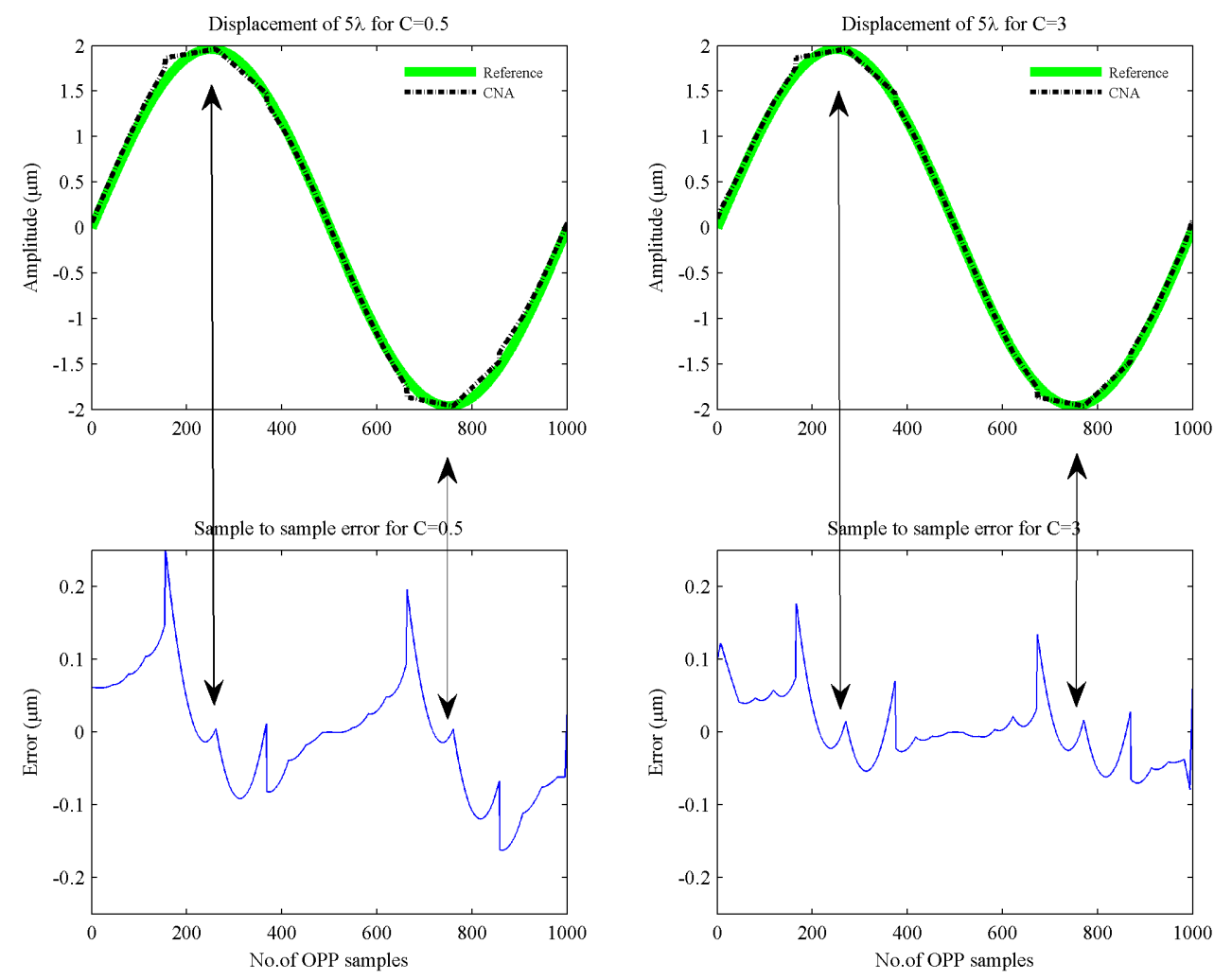

FIGURE 6. Displacement reconstruction and comparative error for two different feedback levels.

i.e. $3 \lambda$ in forward direction and symmetrically backwards. The displacement observed by the PD alongside the transitions from the real signals is shown in Fig.7. The maximum value is $2.32 \mu \mathrm{m}$ and the minimum is $2.3 \mu \mathrm{m}$, that means a total error of $120 \mathrm{~nm}$ per swing.

\section{CONCLUSION}

The presented methodology reduces the problem of SM displacement reconstruction to the efficient usage of an adaptive filter, driven by the number of samples separating two transitions. Its simplicity allows a straightforward estimation of the resources needed to synthesize a given application as well as an implementation maintainability. The precision is kept below $\lambda / 2$, in accordance to the classical reported works. Due to its modularity, it is intended to be coupled to recent research leading to the apparition of robust SM sensors. Notably in terms of robustness against external signal degradations introduced in real scenarios. Its founding basis allows to establish a real time constraint in the development of a system and adapt the algorithm complexity by classical signal processing implementations. The perspective is to integrate this algorithm in different hardware and software architectures in order to provide optimal systems for a wide variety of displacement measurements.

\section{ACKNOWLEDGMENTS}

The first author would like to thank CONACYT for financial support. 

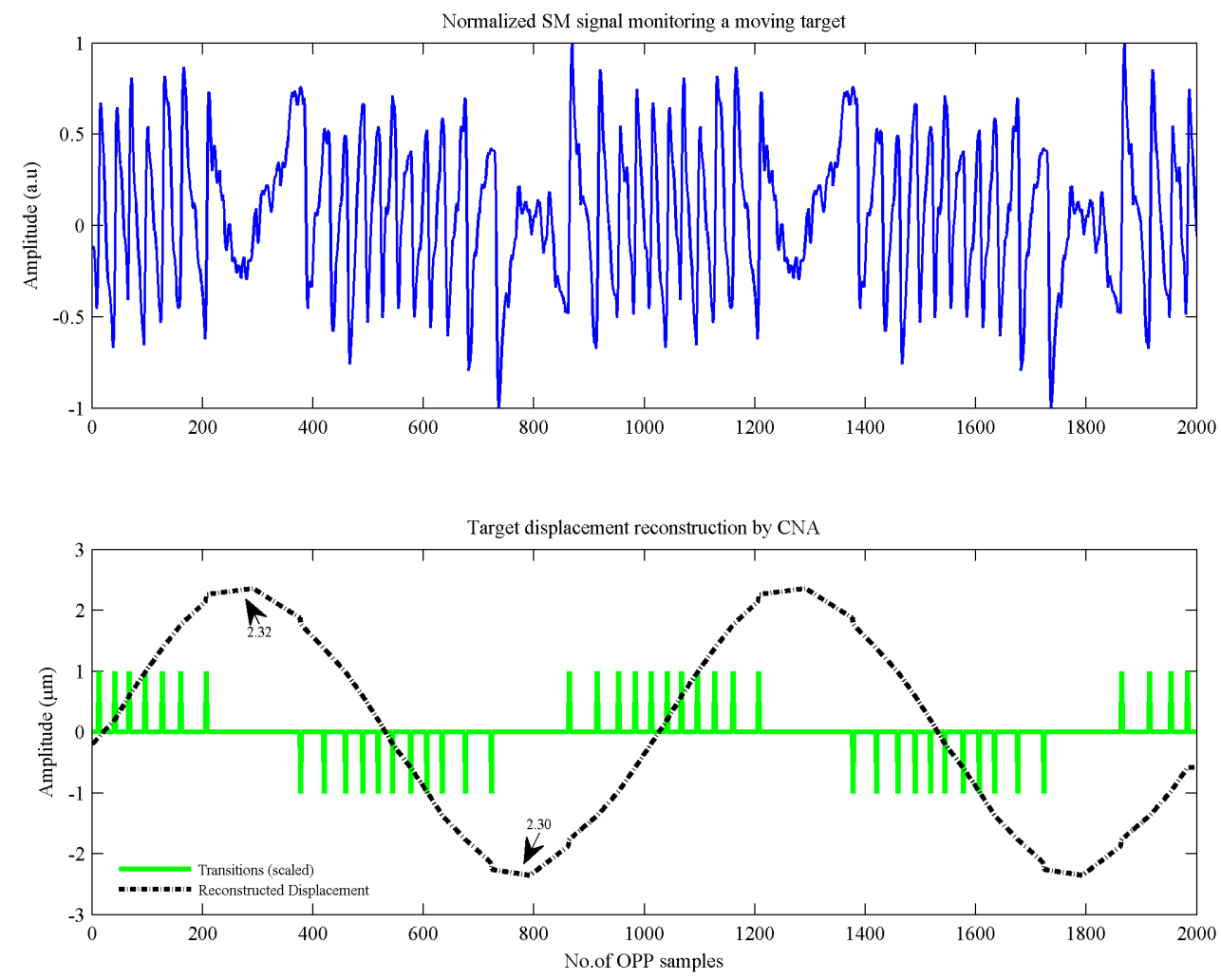

FIGURE 7. SM signal monitoring a piezoelectric in movement and its reconstructed displacement from detected transitions.

\section{REFERENCES}

1. S. Donati, M. Norgia, and G. Giuliani, "A review of self-mixing techniques for sensing applications," in The 17th Annual Meeting of the IEEE Lasers and Electro-Optics Society, 2004, vol. 1, pp. 260 - 261.

2. T. Bosch, C. Bes, L. Scalise, and G. Plantier, "Optical Feedback Interferometry," in Encyclopedia of Sensors, American Scientific Publishers, 2006, vol. 7, pp. 107 - 126.

3. C. Bes, G. Plantier, and T. Bosch, IEEE Trans. Instrum. Meas. 55, $1101-1105$ (2006), ISSN 0018-9456.

4. D. M. Guo, Optics Communications 284, 5766-5769 (2011).

5. Y. L. Fan, Y. G. Yu, J. T. Xi, and J. F. Chicharo, Applied Optics 50, 5064-5072 (2011).

6. F. P. Mezzapesa, L. Columbo, M. Brambilla, M. Dabbicco, A. Ancona, T. Sibillano, F. De Lucia, P. M. Lugara, and G. Scamarcio, Optics Express 19, 16160-16173 (2011).

7. I. Milesi, M. Norgia, P. Pompilio, C. Svelto, and R. Dellaca, IEEE Trans. Instrum. Meas. 60, 2894 -2901 (2011), ISSN 0018-9456.

8. J. G. Proakis, and D. G. Manolakis, Digital Signal Processing - Principles, Algorithms and Applications, Pearson Prentice Hall, 2007.

9. G. Plantier, C. Bes, and T. Bosch, IEEE J. Quantum Electron. 41, 1157 - 1167 (2005), ISSN 0018-9197.

10. S. Maymon, and A. Oppenheim, IEEE Trans. Signal Process. 59, 4745 -4758 (2011), ISSN 1053-587X.

11. C. Cheng, and K. Parhi, IEEE Trans. Circuits Syst. I 51, 1492 - 1500 (2004), ISSN 1549-8328.

12. P-753 LISA Linear Actuator and Stage [Brochure], Physik Instrumente (2008). 